\title{
Metacognitive Therapy for anxiety and depression in cardiac rehabilitation: Commentary on the UK National Institute of Health Research funded PATHWAY programme

\author{
Adrian Wells $\mathbf{s}^{1,2, *}$ \& Cintia Faija ${ }^{1,2}$
}

The University of Manchester, School of Psychological Sciences, Faculty of Biology, Medicine and Health, Oxford Road, Manchester, M13 9PL, United Kingdom ${ }^{2}$ Greater Manchester Mental Health NHS Foundation Trust, Rawnsley Building, Manchester Royal Infirmary, Hathersage Road, Manchester, M13 9WL, United Kingdom

Article Info

\section{Article Notes}

Received: May 08, 2018

Accepted: May 28, 2018

\section{*Correspondence:}

Dr. Adrian Wells, Greater Manchester Mental Health NHS Foundation Trust, Rawnsley Building 2nd Floor, Manchester Royal Infirmary, Hathersage Road, Manchester, M13 9WL, United Kingdom; Email: adrian.wells@manchester.ac.uk

(c) 2018 Wells A. This article is distributed under the terms of the Creative Commons Attribution 4.0 International License.

\section{Keywords}

Cardiac rehabilitation

Anxiety

Depression

Metacognitive therapy

Psychological distress

Psychotherapy

\section{ABSTRACT}

Anxiety and depression are common amongst cardiac patients undergoing cardiac rehabilitation and are associated with poorer health, poor quality of life and higher economic costs. There is a paucity of effective psychological treatments and their provision in this care pathway. A new treatment that is proving highly effective in mental health settings, named metacognitive therapy (MCT), could offer a way forward. This commentary delineates the problem of psychological distress (defined by anxiety and depression symptoms) in cardiac rehabilitation, highlights the limitations of current treatment and describes the PATHWAY research programme funded by the UK National Institute of Health Research (NIHR) to translate and test the metacognitive therapy in this population.

The importance of psychological distress in cardiac patients

Nearly 100,000 patients attend cardiac rehabilitation (CR) services per year in the UK across $324 \mathrm{CR}$ programmes at an annual total cost of approximately $£ 42$ million $^{1-3}$. Data from the UK National Audit of CR (2017) ${ }^{4}$ reported that $28 \%$ of patients attending CR services experience clinical levels of anxiety and 19\% report clinical levels of depression (defined by the Hospital Anxiety and Depression Scale; HADS), highlighting psychological distress is prevalent in this population ${ }^{5-9}$. The rate of major depression identified using structured interviews (across 8 studies) among patients with myocardial infarction was approximately $20 \%{ }^{10-11}$. However, when moderate to significant depressive symptoms were explored (based on self-report measures; across 14 studies) the rate was approximately $40 \%{ }^{10-11}$.

Anxiety and depression in cardiac patients are associated with a range of negative outcomes, such as lower rates of adherence to treatment, a higher prevalence of high-risk behaviors (e.g., smoking), increased risk of death and further cardiac events ${ }^{7,12-16}$. Two recent studies conducted in heart disease patients found that anxiety and depression predicted future symptoms of psychological distress, higher rates of hospital readmissions, greater costs to the health-care system, lower quality of life, and poorer prognosis ${ }^{17-18}$. Unfortunately, there is low effectiveness in managing anxiety and depression in this population which has an impact on the success of $\mathrm{CR}^{19}$. Given the nature of this problem and its impact on the 
individual, on health services and society, it is imperative that anxiety and depression can be effectively treated in CR services.

\section{CR programmes and psychosocial health}

In 2010, the Department of Health implemented a CR commissioning pack ${ }^{20}$. The key aim of the CR programme is to improve physical health and quality of life, and to support people to develop self-management skills. Core components of the patient care plan include health behavior change and education, medical and lifestyle risk factor management (e.g., physical activity, diet, smoking cessation), and psychosocial health. The psychosocial health component is aimed at identifying individuals with clinical levels of anxiety and/or depression, and those with severe and enduring mental health disorders. The second edition of the British Association for Cardiovascular Prevention and Rehabilitation (BACPR) Standards and Core components (2012) ${ }^{21}$ suggests referring these patients to trained psychological practitioners. However, the latest edition $(2017)^{22}$, recommends that when clinical levels of psychological distress are related to the cardiac event, and a trained psychologist practitioner exists in the CR team, the patient could be managed within the service. In the absence of a psychologist in the team, individuals should be referred to trained psychological practitioners and their GP should be informed. It is important to highlight that the majority of CR teams in the UK do not include a psychologist.

Although psychological assessment and support are advocated in the CR commissioning pack $^{20}$ and in other key UK National Health Service policies ${ }^{21-25}$, only $19 \%$ of CR programmes delivered in group format include a psychological component; $2 \%$ of patients receive individual psychotherapy, and no manualized psychological interventions for depression and/or anxiety are available for general use ${ }^{1}$.

Approximately $47 \%$ of cardiac patients experience clinical levels of anxiety or depression at the start of CR but only $12 \%$ move into the normal range after completing the CR programme $6 \%$ for anxiety and $6 \%$ for depression $)^{4}$; thus, most patients continue to experience psychological distress. Furthermore, there is evidence that over time improvements in psychological distress are not maintained ${ }^{4}$. Attempts to treat psychological distress in cardiac patients include a range of different interventions, i.e., counselling, psycho-education, relaxation, stress management, social support, cognitive behavioural therapy (CBT), and motivational interviewing. Psychological interventions have shown non-significant improvements in reducing anxiety and depression ${ }^{25-26}$ or only small effect sizes $^{27-28}$. In addition, pharmacological and psychological treatments in cardiac patients have shown limited effects on health-related quality of life ${ }^{29}$ and no improvement in cardiovascular outcomes ${ }^{30-31}$. We propose that the reason for such approaches having limited effectiveness among cardiac rehabilitation patients could be due to the fact that they focus on changing behaviours or challenging the evidence of negative thoughts (which may be accurate in patients with a physical condition) but they do not directly target the regulation of worry and rumination which play a key role in the maintenance of psychological distress ${ }^{32}$.

Given the limitations of existing treatments in this context, there is a need to develop new and effective psychological treatments that can be implemented in the CR care pathway. The identification of this need has led to a recent research programme, named PATHWAY, funded by the UK NIHR to examine the effects of a psychological treatment called Metacognitive Therapy (MCT) ${ }^{32}$.

\section{What is MCT and why might it be better?}

MCT is grounded in a scientifically supported model of information processing in anxiety and depression ${ }^{33-34}$. It is based on the principle that anxiety and depression result from the activation of a style of excessive and extended thinking dominated by worry, rumination and monitoring for danger. This style increases the sense of current threat. So, in response to a spontaneous negative thought such as "What if I can't work", the person who engages in chains of worrying will prolong and worsen their emotional state. This style of thinking has been found to be linked to underlying metacognitions that are involved in mental regulation ${ }^{32-34}$. MCT focuses on increasing the control of worry and attention in a way that enhances the development of more adaptive metacognitions for self-control.

MCT has been found to be highly effective in mental health settings in the treatment of anxiety and depression and more effective than no-treatment control conditions and $\mathrm{CBT}^{35}$. Unlike treatments such as CBT which challenge patients' negative thoughts, a major advantage of MCT is that patients discover they have control of processes such as worry even when negative thoughts are realistic which is likely to be the case in medical conditions. Furthermore, the set of processes modified in MCT are considered common to most types of psychological distress including acute stress, anxiety, low mood, and grief, meaning a common set of procedures can be applied in a wide group of patients without the need for separate individual formulations. These factors could mean that MCT is more effective than existing psychological approaches when introduced in the CR pathway because it can deal with different types of psychological distress experienced within single groups.

MCT has been found effective in pilot work across a range of medical conditions including cancer ${ }^{36}$, Parkinson disease $^{37-38}$ and chronic fatigue syndrome ${ }^{39-40}$. In addition, a recent systematic review demonstrated that unhelpful 
styles of thinking (worry and/or rumination) predicted depression, anxiety, and psychological distress in people with a range of long-term health conditions including heart disease ${ }^{41}$. Because the control of worry and rumination is specifically tackled in $\mathrm{MCT}^{32}$ this approach could prove to be more helpful.

\section{A brief overview of PATHWAY Work-Streams}

The PATHWAY programme is a 5-year project funded under the UK NIHR Programme Grants for Applied Research award (RP-PG-1211-20011). It is comprised of three work-streams (WS): WS1 $(n=52)$ is a single-blind randomized pilot study comparing Group-MCT plus usual CR (intervention arm) versus usual CR alone (control arm), with 4 and 12 months follow up. WS2 extends data from WS1 with additional recruitment to give an overall sample of 332 and is a full-scale single-blind randomized controlled trial (RCT). WS3 is a feasibility trial $(n=108)$ comparing Home-MCT plus usual CR (intervention arm) versus usual CR (control arm), with 4 and 12 months follow up.

Seven NHS sites located in the North-West of England are involved in the PATHWAY Programme. Sites participating in WS1 include University Hospital of South Manchester NHS Foundation Trust; Central Manchester University Hospitals NHS Foundation Trust and East Cheshire NHS Trust. Sites added to participate in WS2 include Stockport NHS Foundation Trust and Pennine Acute Hospitals NHS Trust. Sites participating in WS3 include Aintree Liverpool University Hospitals NHS Foundation Trust and Bolton NHS Foundation Trust.

Participants for all work-streams are eligible if they are referred to CR services and they report moderate to high levels of psychological distress (defined by a score of 8 or more on the anxiety and/or the depression subscales of the $\mathrm{HADS}^{42}$ ) following a recent cardiac event. The primary outcome in WS1 and WS2 is the HADS at post-treatment (4 months). Secondary outcomes for these two work-streams are 12 month HADS score and a range of other symptoms (e.g., post-traumatic stress) and process measures (e.g., metacognitions). The primary outcome in WS3 is the feasibility of delivering MCT in a home-based format and secondary outcomes include the HADS score and other measures of psychological processes and quality of life at 4 and 12 months follow up. All work-streams incorporate health-economics and qualitative components. Service user involvement is a feature of all aspects of the research programme.

The main trial (WS2) and the home-based intervention (WS3) are registered in clinicaltrials.gov, the identifier is NCT02420431 and NCT03129282 respectively. The full protocol for WS2 has been recently published ${ }^{43}$. In WS2, CR nurses trained in the delivery of manualized MCT will deliver the treatment in groups of patients in addition to usual CR. The group-MCT intervention consists of six weekly sessions over 1-1.5 hours. In WS3, participants will undertake a self-help intervention guided by a treatment manual which contains 6 modules, and CR nurses will provide limited telephone support to offer support and guidance on completing the manual.

The delivery of MCT in these two formats, i.e. group and self-help, includes well-specified techniques for discovering/developing new strategies to regulate worry and rumination, and modify the metacognitive beliefs that maintain unhelpful patterns of thinking

\section{Conclusion}

Provision of effective psychological management of anxiety and depression in CR lacks on a number of levels. One way forward is to develop effective, evidence-based and manualized treatments that can be integrated in the CR pathway. With these aims and objectives, the UK NIHR has funded a programme of research to evaluate the effects of MCT for anxiety and depression in CR patients. This research programme offers a number of important potential impacts. First, we will have valuable data on the effectiveness of MCT applied in this population; second, we will have two structured and manualized interventions (group and self-help); third, we will have information on the longer-term health and economic impact of treatment; finally, we hope to gain valuable knowledge on the conditions required for the implementation and further development of interventions for effectively treating psychological distress in cardiac patients.

It is hoped that the PATHWAY programme will add evidence on MCT among heart disease patients referred to $\mathrm{CR}$ services experiencing psychological distress, which is important to improve clinical outcomes and enhance patient quality of life and cost-effectiveness.

\section{Conflict of interest statement}

Professor Adrian Wells is the developer of MCT and a co-director of the MCT Institute. He is chief investigator and grant holder on the PATHWAY programme. He has authored books on cognitive therapy and metacognitive therapy and receives royalties from these. The authors declare no other conflicts of interest.

\section{Funding information}

The PATHWAY Programme is funded by the UK National Institute for Health Research (NIHR) under its Programme Grants for Applied Research scheme (RP-PG-121120011).

\section{References}

1. National Audit of Cardiac Rehabilitation. Annual Report. York, UK: British Heart Foundation, University of York; 2012. Available from: http://www.cardiacrehabilitation.org.uk/docs/2012.pdf [Accessed 24 May 2018] 
2. NHS Scotland. Cardiac Rehabilitation in Scotland; 2013. Available from: http://www.isdscotland.org/Health-Topics/Heart-Disease/ Publications/ [Accessed 24 May 2018]

3. NHS England. Increase Uptake of Cardiac Rehabilitation for People with Coronary Artery Disease and Following Acute Heart Failure; 2014. Available from: https://www.england.nhs.uk/wp-content/ uploads/2014/02/pm-fs-3-10.pdf [Accessed 24 May 2018].

4. Pogosova N, Saner H, Pedersen SS, et al. Cardiac Rehabilitation Section of the European Association of Cardiovascular Prevention and Rehabilitation of the European Society of Cardiology. European Journal of Preventive Cardiology. 2015; 22(10): 1290-306.

5. Gandjour A, Lauterbach KM. Review of quality-of-life evaluations in patients with angina pectoris. Pharmacoeconomics. 1999; 16(2): 141-52.

6. Lewin RJP. Improving quality of life in patients with angina. Heart. 1999; 82: 654-655.

7. Naylor C, Parsonage M, McDaid D, et al. Report. Long-term conditions and mental health - The cost of co- morbidities. The King's Fund and Centre for Mental Health. 2012; 1: 32. Available from: https://www. kingsfund.org.uk/publications/long-term-conditions-and-mentalhealth [Accessed 24 May 2018].

8. Thombs BD, Bass EB, Ford DE, et al. Prevalence of depression in survivors of acute myocardial infarction - Review of the Evidence. Journal of General Internal Medicine. 2006; 21(1): 30-38.

9. Tully PJ, Cosh SM, Baumeister H. The anxious heart in whose mind? A systematic review and meta-regression of factors associated with anxiety disorder diagnosis, treatment and morbidity risk in coronary heart disease. Journal of Psychosomatic Research. 2014; 77(6): 43948.

10. Thombs BD, Bass EB, Ford DE, et al. Prevalence of depression in survivors of acute myocardial infarction. Journal of General Internal Medicine. 2006; 21(1): 30-38.

11. Carney RM, Freedland KE. Depression, mortality, and medical morbidity in patients with coronary heart disease. Biological Psychiatry. 2003; 54(3): 241-247.

12. Bush DE, Ziegelstein RC, Tayback M, et al. Even minimal symptoms of depression increase mortality risk after acute myocardial infarction. American Journal of Cardiology. 2001; 88(4): 337-41.

13. Hare DL, Toukhsati SR, Johansson P, et al. Depression and cardiovascular disease: A clinical review. European Heart Journal. 2014; 35(21): 1365-72.

14. Rutledge T, Linke SE, Krantz DS, et al. Psychosomatic Medicine. 2009; 71(9): 958-64.

15. Batelaan NM, Seldenrijk A, Bot M, et al. Anxiety and new onset of cardiovascular disease: Critical review and meta-analysis. British Journal of Psychiatry. 2016; 208(3): 223-31.

16. Frasure-Smith N, Lespérance F, Talajic M. Depression and 18-month prognosis after myocardial infarction. Circulation. 1993; 91(4): 9991005.

17. Palacios J, Khondoker M, Mann A, et al. Depression and anxiety symptom trajectories in coronary heart disease: Associations with measures of disability and impact on 3-year health care costs. Journal of Psychosomatic Research. 2018; 104: 1-8.

18. Baumeister $\mathrm{H}$, Haschke $\mathrm{A}$, Munzinger $\mathrm{M}$, et al. Inpatient and outpatient costs in patients with coronary artery disease and mental disorders: A systematic review. BioPsychoSocial Medicine. 2015; 17: 9-11.

19. National Audit of Cardiac Rehabilitation (NACR) - Annual Statistical Report 2017. Available from https://www.bhf.org.uk/publications/ statistics/national-audit-of-cardiac-rehabilitation-annual-statisticalreport-2017. [Accessed 24 May 2018]
20. Department of Health. Commissioning Pack for Cardiac Rehabilitation. London, UK: Department of Health; 2010. Available from: http://webarchive.nationalarchives.gov.uk/+/ www.dh.gov.uk/en/Publicationsandstatistics/Publications/ PublicationsPolicyAndGuidance/Browsable/DH_117504 [Accessed 24 May 2018]

21. British Association for Cardiovascular Prevention and Rehabilitation. The BACPR Standards and Core Components for Cardiovascular Disease Prevention and Rehabilitation ( $2^{\text {nd }}$ Edition). UK: BACPR; 2012. Available from: http://www.bacpr.com/resources/46C_BACPR_ Standards_and_Core_Components_2012.pdf [Accessed 24 May 2018]

22. British Association for Cardiovascular Prevention and Rehabilitation. The BACPR Standards and Core Components for Cardiovascular Disease Prevention and Rehabilitation (3 ${ }^{\text {rd }}$ Edition). UK: BACPR; 2017. Available from: http://www.bacpr.com/resources/BACPR Standards_and_Core_Components_2017.pdf [Accessed 24 May 2018]

23. HM Government. No Health without Mental Health: A CrossGovernment Mental Health Outcomes Strategy for People of All Ages. London, UK: Department of Health; 2011. Available from: https:// www.gov.uk/government/uploads/system/uploads/attachment_ data/file/213761/dh_124058.pdf [Accessed 24 May 2018]

24. National Institute for Health and Clinical Excellence. Depression in Adults with a Chronic Physical Health Problem: Recognition and Management. London: National Institute for Health and Clinical Excellence; 2009. Available from: https://www.nice.org.uk/ Guidance/CG91 [Accessed 624 May 2018]

25. Reid J, Ski CF, Thompson DR. Psychological interventions for patients with coronary heart disease and their partners: A systematic review. PloS ONE. 2013; 8(9): e73459.

26. Jiang Y, Shorey S, Seah B, et al. The effectiveness of psychological interventions on self-care, psychological and health outcomes in patients with chronic heart failure - A systematic review and metaanalysis.International Journal of Nursing Studies. 2018; 78: 16-25.

27. Whalley B, Rees K, Davies P, et al. Psychological interventions for coronary disease (Review). Cochrane Database Systematic Reviews. 2011; (8): CD002902.

28. Dickens C, Cherrington A, Adeyemi I, et al. Characteristics of psychological interventions that improve depression in people with coronary heart disease: A systematic review and meta-regression. Psychosomatic Medicine. 2013; 75(2): 211-221.

29. O'Neil A, Sanderson K, Oldenburg B, et al. Impact of depression treatment on mental and physical health-related quality of life of cardiac patients: A meta-analysis. Journal of Cardiopulmonary Rehabilitation and Prevention. 2011; 31(3): 146-56.

30. Baumeister H, Hutter N, Bengel J. Psychological and pharmacological interventions for depression in patients with coronary artery disease. Cochrane Database Systematic Reviews. 2011; (9): CD008012.

31. Rutledge T, Redwine L, Linke SE, et al. A meta-analysis of mental health treatments and cardiac rehabilitation for improving clinical outcomes and depression among patients with coronary heart disease. Psychosomatic Medicine. 2013; 75(4): 335-349.

32. Wells A. Metacognitive Therapy for Anxiety and Depression. London, UK; Guilford Press; 2009.

33. Wells A, Matthews G. Attention and emotion: A clinical perspective. Hove:Erlbaum; 1994.

34. Wells A, Matthews G. Modelling Cognition in Emotional Disorder: The S-REF MODEL. Behaviour Research Therapy. 1996; 34(11): 881-888.

35. Normann N, van Emmerik AAP, Morina N. The efficacy of metacognitive therapy for anxiety and depression: A meta-analytic review. Depression and Anxiety. 2014; 31(5): 402-411.

36. Cook SA, Salmon P, Dunn G, et al. A prospective study of the association 
of metacognitive beliefs and processes with persistent emotional distress after diagnosis of cancer. Cognitive Therapy and Research. 2015; 39(1): 51-60.

37. Allott R, Wells A, Morrison AP, et al. Distress in Parkinson's disease: Contributions of disease factors and metacognitive style. British Journal of Psychiatry. 2005; 187(2): 182-183.

38. Fernie BA, Spada MM, Ray Chaudhuri K, et al. Thinking about motor fluctuations: An examination of metacognitions in Parkinson's disease. Journal of Psychosomatic Research. 2015; 79(6): 669-73.

39. Maher-Edwards L, Fernie BA, Murphy G, et al. Metacognitions and negative emotions as predictors of symptom severity in chronic fatigue syndrome. Journal of Psychosomatic Research. 2011; 70(4): 311-7.
40. Maher-Edwards L, Fernie, BA, Murphy G, et al. Metacognitive factors in chronic fatigue syndrome. Clinical Psychology and Psychotherapy. 2012; 19(6): 552-7.

41. Trick $\mathrm{L}$, Watkins $\mathrm{E}$, Windeatt $\mathrm{S}$, et al. The association of perseverative negative thinking with depression, anxiety and emotional distress in people with long term conditions: A systematic review. Journal of Psychosomatic Research. 2016; 91: 89-101.

42. Zigmond AS, Snaith RP. The hospital anxiety and depression scale. Acta Psychiatrica Scandinavica. 1983; 67(6): 361-370.

43. Wells A, McNicol K, Reeves D, et al. Improving the effectiveness of psychological interventions for depression and anxiety in the cardiac rehabilitation pathway using group-based metacognitive therapy (PATHWAY Group MCT): Study protocol for a randomised controlled trial. Trials. 2018; 19: 215. 